\title{
Inmigración masiva, salarios reales y empleo: un modelo keynesiano
}

\author{
Oscar DANCOURT
}

\begin{abstract}
RESUMEN
La llegada masiva de los inmigrantes venezolanos, que escapan de la catástrofe macroeconómica causada por el gobierno de Maduro, es un evento único en la historia económica reciente del Perú. Este texto intenta mostrar que un modelo keynesiano, donde el empleo depende de la demanda agregada, puede identificar los dos canales a través de los cuales una inmigración masiva reduce, en el corto plazo, el empleo y los salarios reales. Estos dos canales son el incremento de la fuerza laboral que reduce los salarios nominales y reales, y la disminución de la propensión a consumir de los asalariados si ocurre una sustitución de trabajadores locales por inmigrantes entre los ocupados.

Los trabajadores venezolanos no tienen responsabilidad alguna en la creación de esta situación. Como los peruanos que han emigrado desde la década de 1980, ellos solo buscan lo mejor para sus familias. La responsabilidad entera la tiene el gobierno de Kuczynski y su política de libre inmigración que amplia artificialmente el excedente de mano de obra urbana existente en la economía peruana.
\end{abstract}

Palabras clave: inmigración, salario real, empleo, modelos keynesianos.

Clasificación JEL: E240, E120, J610

\section{Mass immigration, real wages and employment: a Keynesian model}

\begin{abstract}
The mass arrival of Venezuelan immigrants escaping the macroeconomic catastrophe caused by the Maduro government is a unique event in Peru's recent economic history. This text uses a Keynesian model, where employment depends on aggregate demand, in an attempt to identify the two channels through which mass immigration reduces, in the short term, employment and real wages. These two channels are the increase in the labor force, which reduces nominal and real wages; and the decrease in employees' propensity to consume if immigrants substitute local workers in the labor market.
\end{abstract}

* Profesor principal del Departamento de Economía de la PUCP. 
Venezuelan workers bear no responsibility whatsoever for bringing about this situation. Like the many Peruvians who have emigrated since the 1980s, they only look for the best for their families. All of the responsibility lies with the Kuczynski government and its policy of free immigration, which artificially extends the surplus of urban labor in the Peruvian economy.

Keywords: immigration, employment, real wages, Keynesian models.

JEL classification: E240, E120, J610

\section{INTRODUCCIÓN}

La economía peruana tuvo una inflación más baja y creció más rápido que casi cualquier otra de América del Sur, desde principios de los años 2000 hasta 2013. Este desempeño macroeconómico nunca fue suficiente, sin embargo, para atraer mano de obra de los países vecinos. Porque aquí hay un gran excedente de mano de obra urbana, definido como la fuerza laboral total menos los que trabajan en el sector moderno (empresas con 10 y más trabajadores), que mantiene bajos los salarios. Este excedente de mano de obra gira todavía alrededor del $65 \%$ de la fuerza laboral urbana, a pesar de esa década y pico de rápido crecimiento económico. Por eso, desde la década de 1980, el Perú es un país de emigrantes (véase Altamirano, 2010).

La llegada masiva de los inmigrantes venezolanos, que escapan como pueden de la catástrofe macroeconómica causada por el gobierno de Maduro, es un evento único en la historia económica reciente del Perú. La hiperinflación en Venezuela ha reducido enormemente los salarios reales y, para colmo de males, viene acompañada de una gran recesión, una combinación letal similar a la que sufrimos los peruanos a fines de la década de 1980 durante el primer gobierno de Alan García. Esta es la fuerza básica que genera el éxodo venezolano dirigido a cualquier lugar donde haya mejores salarios y oportunidades de empleo. El otro factor clave, que explica por qué el destino de los trabajadores venezolanos es el Perú y no cualquier otro país vecino con similar estabilidad macroeconómica, es la política de libre inmigración aplicada por el gobierno de Kuczynski. Esta política está diseñada para fomentar esta inmigración venezolana, sin límite cuantitativo alguno, como ha argumentado Freyre Paulet (2018).

El problema es que la inmigración masiva reduce los salarios y aumenta el desempleo, como atestigua la literatura especializada; véase Hatton y Williamson (2005) y Borjas y Aydemir (2006). Pero, los abogados defensores de esta política de libre inmigración del gobierno de Kuczynski sostienen que el aumento de la fuerza laboral generado por esta inmigración masiva no causará perjuicio alguno a los trabajadores locales.

Un botón de muestra extraído de la literatura especializada. Cuando Argelia se independizó de Francia en 1962, retornaron 900 mil personas al país europeo en un año. 
La fuerza laboral francesa aumento en 1,6 por ciento por esta repatriación de mano de obra calificada. Un estudio posterior (Hunt, 1992) con datos censales encontró que un incremento de un punto porcentual en el peso que estos repatriados tenían en la fuerza laboral de una región cualquiera de Francia, reducía el salario de esa región en 0,5-0,8 de un punto porcentual y elevaba la tasa de desempleo en $0,1-0,3$ de un punto porcentual entre los trabajadores locales de esa región.

En el Perú, la fuerza laboral urbana (población económicamente activa) era de 13 millones de personas en 2015, según el INEI. Y la mitad, 6 millones y medio, tenían entre 15 y 39 años, que debe ser el rango de edad de los inmigrantes venezolanos, que difícilmente se convertirán en campesinos. Si son 130 mil los inmigrantes venezolanos que van a ingresar a la fuerza laboral urbana, estos representarían un 2 por ciento de la PEA entre 15 y 39 ańos y un 1 por ciento de la fuerza laboral urbana total. Si son 260 mil estos inmigrantes, representarían entonces un 4 por ciento de la PEA entre 15 y 39 años o un 2 por ciento de la fuerza laboral urbana total.

Sería un milagro que este repentino incremento de la fuerza laboral urbana no provoque una reducción del salario promedio y un aumento de la informalidad y el desempleo, especialmente si el sindicalismo es débil y si la economía del Perú urbano está paralizada desde hace cuatro años. El empleo en el sector moderno (empresas de 10 y más trabajadores), que solo representa alrededor del 35 por ciento de la fuerza laboral urbana, ha caído ininterrumpidamente entre julio y noviembre pasados, según el Ministerio de Trabajo.

Se podría discutir también cuál sería el impacto de esta inmigración masiva sobre el consumo agregado de los trabajadores ocupados. Un punto a tener en cuenta es que la mayoría de las familias de estos trabajadores inmigrantes debe estar todavía en Venezuela. Eso implica que a igual salario, un trabajador inmigrante consumirá menos que un trabajador local, y ahorrará más, para poder enviar dinero a su familia.

El objetivo de este texto es mostrar que un modelo macroeconómico keynesiano, donde el empleo total depende de la demanda agregada, puede identificar claramente los dos canales a través de los cuales una inmigración masiva reduce, en el corto plazo, el empleo y los salarios reales. Estos dos canales son el incremento de la fuerza laboral que reduce los salarios nominales y reales, y la disminución de la propensión a consumir de los asalariados si ocurre una sustitución de trabajadores locales por inmigrantes entre los ocupados.

Para terminar esta introducción, quiero enfatizar que los trabajadores venezolanos no tienen responsabilidad alguna en la creación de esta situación. Como los peruanos que han emigrado desde la década de 1980, ellos solo buscan lo mejor para sus familias. La responsabilidad entera la tiene el gobierno de Kuczynski y su política de libre inmigración que amplía artificialmente el excedente de mano de obra urbana existente en la economía peruana. 


\section{EMPLEO, SALARIOS Y PRECIOS}

El primer componente del modelo se refiere a las funciones de producción y a la composición de la mano de obra. Hay dos sectores productivos en esta economía (bien final e insumo) y dos tipos de mano de obra (local e inmigrante). Las funciones de producción de corto plazo son

(1) $Y_{F}=a_{F} L_{F}$

(2) $Y_{I}=a_{I} L_{I}$

(3) $L=L_{F}+L_{I}$

donde $Y_{F}, Y_{I}$ es la producción total en cada industria, $a_{F}, a_{I}$ es el producto por trabajador en cada industria, y $L_{F}, L_{I}$ es el empleo en cada sector; el subíndice $\mathrm{F}$ indica la industria del bien final y el subíndice I la industria del insumo. Si todos los trabajadores ocupados tienen la misma jornada laboral podemos medir el empleo en horas trabajadas o en número de personas ocupadas. El empleo total (L) es la suma del empleo en ambas industrias.

Las ecuaciones (4) y (5) establecen que hay dos tipos de trabajadores ocupados, los locales (subíndice 1) y los inmigrantes (subíndice 2), en proporciones $\theta$ y $1-\theta$, respectivamente, del empleo total.

(4) $L_{1}=\theta L$

(5) $L_{2}=(1-\theta) L$

La ecuación (6) determina el excedente de mano de obra ${ }^{1}$ o desempleo (D), que depende de la fuerza laboral $\left(\mathrm{L}^{*}\right)$ y del empleo agregado (L). Es decir,

(6) $D=L^{*}-L$

El segundo componente del modelo se refiere a las funciones de demanda del bien final y del insumo. La demanda agregada del bien final está compuesta por el consumo (C) y la inversión (I). El consumo, a su vez, solo depende del salario real (W/P) y del empleo total; los beneficios se ahorran íntegramente. La propensión a consumir es uno para los trabajadores locales $\left(L_{1}\right)$ y $c_{2}$, que está entre cero y uno, para los trabajadores inmigrantes $\left(L_{2}\right)$ que deben ahorrar (como destaca Borjas (2009), remesan una parte de sus salarios al exterior). Es decir,

(7) $Y_{F}^{d}=C+I$

1 El excedente de mano de obra en el Perú urbano incluye a los desempleados y a los informales (autoempleados o trabajadores independientes) que preferirían trabajar en el sector moderno si pudieran. Aquí se asume que la informalidad no existe y que el desempleo es igual al excedente de mano de obra. Para una discusión sobre cómo se distribuye el excedente de mano de obra entre desempleo e informalidad, véase Dancourt (1990). 
(8) $C=\frac{W}{P} L_{1}+\frac{c_{2} W}{P} L_{2}$

La función de inversión es la del libro de texto. La inversión tiene un componente autónomo $\left(I_{0}\right)$ que representa el optimismo/pesimismo de los empresarios y también depende inversamente del costo del crédito (i). Es decir,

(9) $I=I_{0}-b i$

La ecuación (10) establece que la demanda del insumo depende de la producción del bien final, donde h es la cantidad de insumo por unidad de bien final. Es decir,

(10) $Y_{I}^{d}=h Y_{F}$

El tercer componente del modelo se refiere a las ecuaciones de precios (11) y salarios (12). El precio del bien final $(P)$ depende de sus costos de mano de obra $\left(W / a_{F}\right)$ e insumos $\left(h P_{I}\right)$ y de un mark-up $(z)$. El precio del insumo (servicio eléctrico) es una variable exógena, un precio regulado. Es decir,

(11) $P=(1+z)\left(\frac{W}{a_{F}}+h P_{I}\right)$

Y el salario nominal $(W)$ depende inversamente del desempleo $\left(D=L^{*}-L\right)$, esto es, directamente del empleo $(L)$ e inversamente de la fuerza laboral $\left(L^{*}\right)$. Y también depende directamente de un componente autónomo $\left(W_{0}\right)$, como el salario mínimo legal. Es decir,

(12) $W=F_{1}\left(W_{0} ; L ; L^{*}\right)$

\section{DEMANDA Y OFERTA AGREGADAS.}

El modelo puede resumirse en dos ecuaciones que conectan el salario real y el empleo total. La primera ecuación es la demanda agregada (DA) que se obtiene a partir de la condición producción igual demanda (consumo más inversión) del bien final. Es decir,

(13) $Y_{F}=Y_{F}^{d}=C+I$

Insertando las ecuaciones (1), (8) a (10), (4) y (5), podemos reescribir esta condición producción igual demanda como

(14) $a_{F} L_{F}=\frac{W}{P} \theta L+\frac{c_{2} W}{P}(1-\theta) L+I_{0}-b i$ 
Igualando la producción $\left(Y_{i}\right)$ y la demanda $\left(h Y_{F}\right)$ del insumo, e insertando las ecuaciones (1) a (3), tenemos

(15) $L=\left(1+\frac{h a_{F}}{a_{I}}\right) L_{F}$

Si suponemos, para simplificar la notación, que $a_{F}=a_{I}=h=1$, de (15) resulta que $L=2 L_{F}$. De (14) y (15), con estos valores ${ }^{2}$, obtenemos la siguiente relación entre el salario real y el empleo que denominamos demanda agregada (DA).

(16) $\frac{1}{2} L=\frac{W}{P} \theta L+\frac{c_{2} W}{P}(1-\theta) L+I_{0}-b i$

Es decir,

(DA) $L=k\left(I_{0}-b i\right)$

donde el multiplicador keynesiano (k) está dado por

(17) $k=\frac{1}{\frac{1}{2}-c \frac{W}{P}}$

Según (DA), el empleo agregado (L) depende directamente de la inversión privada y del multiplicador keynesiano (k), si existe capacidad productiva ociosa y desempleo. Este multiplicador ${ }^{3}$ es mayor mientras mayor sea el salario real (W/P), porque el consumo se eleva con este salario, dado el empleo agregado. Esto explica la relación directa entre salario y empleo de la ecuación (DA) que se representa en el gráfico 1. La curva DA seria completamente vertical si la función consumo no dependiera del salario real, como ocurre en el libro de texto ${ }^{4}$.

Este multiplicador keynesiano también depende directamente de la propensión media a consumir de los trabajadores (c), que es un promedio ponderado de la propensión a consumir de los trabajadores locales (que hemos supuesto igual a 1) y de la propensión a consumir de los trabajadores inmigrantes $\left(c_{2}\right)$ que hemos supuesto es inferior a 1; donde las ponderaciones reflejan la participación de los trabajadores locales $(\theta)$

2 Ambos productos por trabajador así como el coeficiente de insumo por unidad de bien final reflejan la técnica imperante y, al igual que el stock de capital del cual dependen, se consideran constantes en el corto plazo. 3 Si hay beneficios en ambas industrias, el multiplicador es positivo. Para que esto ocurra, se requiere que $P>c 2 W$. Suponiendo $a_{F}=a_{I}=h=1$, el precio del bien final es $P=(1+z)\left(W+P_{I}\right)$ y el precio del insumo es $P_{I}=W+G_{I}$, donde $G_{I}$ es la ganancia por unidad de insumo producido. Es decir, $P=(1+z)\left(2 W+G_{I}\right)$. Por tanto, $P>c 2 W$ si $(1+z)\left(1+G_{I} / 2 W\right)>c$, lo que se cumple siempre que $G_{I}$ sea positivo pues c es menor que uno. Las ganancias por unidad de producto en la producción del bien final son positivas si el mark-up $(z)$ es mayor que cero.

4 En este modelo, la curva DA tiene pendiente negativa si la propensión a consumir (menor que uno) es la misma para asalariados y capitalistas, como en el libro de texto. 
e inmigrantes $(1-\theta)$ en el empleo total. Es decir, $c=\theta+(1-\theta) c_{2}$. Por tanto, mientras menor sea $\theta$, la participación de los trabajadores locales en el empleo total, menor será la propensión media a consumir y menor será el multiplicador keynesiano.

En el grafico 1, la curva DA se desplaza hacia la izquierda si $\theta$ disminuye con la libre inmigración porque se reduce la propensión media a consumir (c) de los asalariados.

La oferta agregada (OA) es la segunda ecuación que resume el modelo y conecta los salarios reales con el empleo. Esta ecuación la obtenemos de las ecuaciones de precios (11) y salarios (12) y de la definición del salario real en términos del bien final (W/P).

El salario real $(\mathrm{W} / \mathrm{P})$ es una función directa del salario nominal $(\mathrm{W})$ e inversa del precio del insumo $\left(P_{I}\right)$ y del mark-up $(z)$. Si suponemos que $a_{F}=a_{I}=h=1$, tenemos que

(18) $\frac{W}{P}=\frac{W}{(1+z)\left(W+P_{I}\right)}=\frac{1}{(1+z)\left(1+P_{I} / W\right)}$

Que, podemos expresar así

(19) $\frac{W}{P}=F_{2}\left(W ; P_{I} ; z\right)$

Y como el salario nominal $(W)$ depende inversamente del desempleo según (12), entonces, resulta que el salario real también depende directamente del empleo $(L)$ e inversamente de la fuerza laboral $\left(L^{*}\right)$. Es decir, la ecuación de oferta agregada $(\mathrm{OA})$ es

(OA) $\frac{W}{P}=F_{3}\left(L ; L^{*} ; W_{0} ; P_{I} ; z\right)$

La ecuación (OA), que también establece una relación directa entre el empleo agregado y el salario real, se presenta en el gráfico 1. Como en el libro de texto, cuando el empleo aumenta, se reduce el desempleo, mejora la capacidad de negociación de los trabajadores, se eleva el salario nominal y, dado el precio del insumo y el mark-up, también se eleva el salario real. La curva OA se desplaza hacia la derecha si la fuerza laboral $\left(L^{*}\right)$ aumenta con la libre inmigración porque aumenta el desempleo y se reducen el salario nominal y el salario real, para un mismo nivel de empleo. El efecto es similar al de una rebaja del salario mínimo legal $\left(W_{0}\right)$.

La intersección de la oferta y la demanda agregadas determina el salario real y el nivel de empleo agregado. Para que el modelo sea estable se requiere que la pendiente de la curva DA sea mayor que la pendiente de la curva OA. Esto es, se requiere ${ }^{5}$ que

(20) $\frac{1}{R}>\left(1-\delta_{3}\right) \delta_{1}$

\footnotetext{
5 Véase el Apéndice 1.
} 


\section{Gráfico 1}

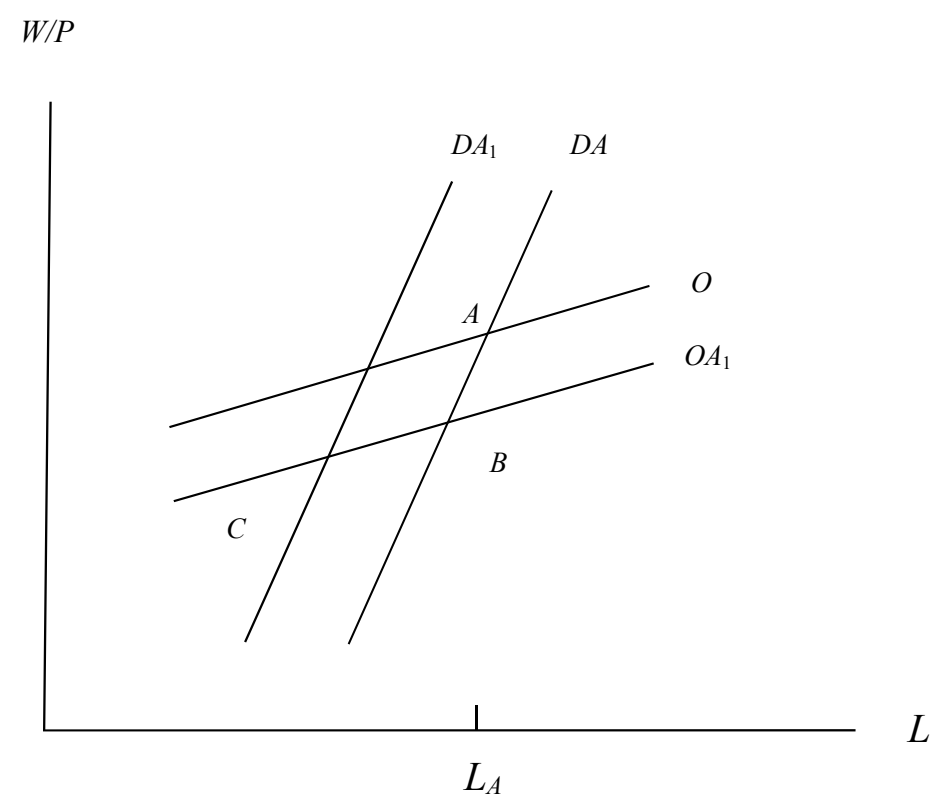

Es decir, requerimos que la inversa de la elasticidad del empleo respecto al salario real $(\mathrm{R})$, factor que define la pendiente de la curva de demanda agregada, sea mayor que el producto de la elasticidad del salario nominal respecto al empleo $\left(\delta_{1}\right)$ y uno menos la elasticidad del precio del bien final respecto al salario nominal $\left(1-\delta_{3}\right)$, factores que definen la pendiente de la curva de oferta agregada. Si R es igual o menor que uno, dado que $\delta_{3}$ es menor que uno, bastaría que la sensibilidad del salario nominal al empleo sea reducida ${ }^{6}$ para que el modelo sea estable; esto hace que la curva OA sea menos empinada que la curva DA, como ocurre en el gráfico 1.

Si la libre inmigración provoca un aumento de la fuerza laboral $\left(L^{*}\right)$, eso traslada hacia abajo la curva de oferta agregada, desde OA hasta $\mathrm{OA}_{1}$. Se reduce el salario real y el empleo agregado. Vamos del punto A al punto B. La cadena causal se inicia con el aumento del desempleo debido a la mayor fuerza laboral, que presiona a la baja el salario nominal y también el salario real. Esto disminuye el consumo agregado y hace caer la producción y el empleo.

Si la libre inmigración provoca, además, una sustitución de trabajadores locales por inmigrantes entre los ocupados, entonces se reducirá también la propensión media a consumir (c), lo que causará una caída del consumo agregado, la producción y el empleo. La curva DA se desplaza hacia la izquierda desde DA hasta $\mathrm{DA}_{1}$. Vamos del punto $\mathrm{B}$ al punto $\mathrm{C}$.

\footnotetext{
6 En la literatura referida a la curva de salarios, la elasticidad del salario nominal a la tasa de desempleo gira alrededor de -0.1; véase Blanchflower y Posen (2014).
} 


\section{A MODO DE CONCLUSIÓN}

Por ambos canales, vía su impacto en el excedente de mano de obra y en la propensión media a consumir de los asalariados, una inmigración masiva causa en el corto plazo una caída de los salarios reales y el empleo. El nivel de precios también debería descender como consecuencia de una inmigración masiva, si los precios de los insumos, el mark-up y el salario mínimo no varían.

Supongamos que el banco central aplica una regla de Taylor y baja la tasa de interés (i) cuando cae el nivel de precios $(\mathrm{P})$ o cuando aumenta el desempleo $\left(L^{*}-L\right)$. Ante un episodio de inmigración masiva, el banco central debiera bajar la tasa de interés porque cae el nivel de precios y aumenta el desempleo. En el Grafico 1, una rebaja de la tasa de interés traslada la curva $\mathrm{DA}_{1}$ hacia la derecha. Si la rebaja de la tasa de interés es suficiente (y posible) podemos retornar hasta la curva DA original, o ir más a la derecha, para alcanzar el empleo inicial $\left(L_{\mathrm{A}}\right)$ sobre la nueva curva de oferta agregada $\left(\mathrm{OA}_{1}\right)$. Sin embargo, el salario real sería inferior al del punto original A porque la fuerza laboral es mayor. Esta política monetaria expansiva solo podría cancelar una parte de los efectos perjudiciales de la inmigración masiva en el corto plazo.

\section{APÉNDICE 1}

Diferenciando totalmente la ecuación (DA), y dividendo ambos miembros entre $L$, se obtiene que

$$
\text { (DA) } \frac{d L}{L}=\frac{d k}{k}+\frac{d I}{I}
$$

Es decir, que el cambio porcentual en el empleo $(d L / L)$ es igual al cambio porcentual en el multiplicador $(\mathrm{dk} / \mathrm{k})$ más el cambio porcentual en la inversión privada $(d I / I)$.

Diferenciando totalmente la ecuación (17) que define el multiplicador, manteniendo constante la propensión a consumir $(c)$, dividiendo ambos miembros entre $k$, y dividiendo y multiplicando el miembro derecho por $(W / P)$, se obtiene que

$$
\text { (17) } \frac{d k}{k}=\frac{\frac{c W}{P}}{\frac{1}{2}-\frac{c W}{P}} \frac{d \frac{w}{p}}{\frac{w}{p}}=R \frac{d \frac{w}{p}}{\frac{w}{p}}
$$

Esto es, que el cambio porcentual en el multiplicador $(d k / k)$ es igual a $R$ veces el cambio porcentual en el salario real $[d(w / p) /(w / p)]$. 
Haciendo el cambio porcentual de la inversión privada igual a cero, de (DA) y (17), obtenemos que

$$
\text { (DA) } \frac{d L}{L}=R \frac{d \frac{w}{p}}{\frac{w}{p}}
$$

Es decir, que el cambio porcentual en el empleo es igual a $\mathrm{R}$ veces el cambio porcentual en el salario real. $\mathrm{R}$ es la elasticidad del empleo agregado ante cambios en el salario real. Si la participación de los salarios directos en el valor bruto de producción del bien final $\left(W L_{F} / P Y_{F}\right)$ oscila entre un quinto y un tercio, y la propensión a consumir es 0.75 , entonces $\mathrm{R}$ debe tener un valor entre 0.5 y 1 . La pendiente de la curva DA en el gráfico 1 está dada por $(1 / \mathrm{R})(\mathrm{w} / \mathrm{p}) / \mathrm{L}$.

Si diferenciamos totalmente la ecuación (11) del precio del bien final, manteniendo constante el mark-up y el precio del insumo, y dividimos ambos miembros entre $\mathrm{P}$, obtenemos que

(11) $\frac{d P}{P}=\frac{W}{W+P_{I}} \frac{d W}{W}=\delta_{3} \frac{d W}{W}$

Esto es, que el cambio porcentual del precio del bien final $(\mathrm{dP} / \mathrm{P})$ es igual a $\delta_{3}$ veces el cambio porcentual en el salario nominal $(d W / W)$; donde $\delta_{3}$, que representa el peso de los costos de mano de obra en la estructura de costos del bien final, es menor que uno.

De la definición del salario real obtenemos que la variación porcentual del salario real es igual a la variación porcentual del salario nominal menos la variación porcentual del nivel de precios. Es decir,

$$
\text { (21) } \frac{d \frac{w}{p}}{\frac{w}{p}}=\frac{d W}{W}-\frac{d P}{P}
$$

Por último, si diferenciamos totalmente la ecuación (12) del salario nominal $(W)$, manteniendo constante el salario mínimo legal $\left(W_{0}\right)$, obtenemos que

(12) $d W=F_{2}{ }^{L} d L-F_{2}{ }^{*} d L^{*}$

Es decir,

$$
\text { (12) } \frac{d W}{W}=\frac{L F_{2}^{L}}{W} \frac{d L}{L}-\frac{L^{*} F_{2}^{L^{*}}}{W} \frac{d L^{*}}{L^{*}}=\delta_{1} \frac{d L}{L}-\delta_{2} \frac{d L^{*}}{L^{*}}
$$

Esto es, que el cambio porcentual del salario nominal $(d W / W)$ es igual a $\delta_{1}$ veces el cambio porcentual en el empleo $(d L / L)$ menos $\delta_{2}$ veces el cambio porcentual en la 
fuerza laboral $\left(d L^{*} / L^{*}\right) ; \delta_{1}$ y $\delta_{2}$ son las elasticidades del salario nominal ante un cambio del empleo y de la fuerza laboral, respectivamente.

De las ecuaciones (11), (12) y (20), obtenemos la ecuación de la oferta agregada.

$$
\left(\mathrm{OA} \frac{d \frac{w}{p}}{\frac{w}{p}}=\left(1-\delta_{3}\right)\left(\delta_{1} \frac{d L}{L}-\delta_{2} \frac{d L^{*}}{L^{*}}\right)\right.
$$

Es decir, que el cambio porcentual en el salario real es igual a $\left(1-\delta_{3}\right) \delta_{1}$ veces el cambio porcentual en el empleo agregado $(d L / L)$ menos $\left(1-\delta_{3}\right) \delta_{2}$ veces el cambio porcentual en la fuerza laboral $\left(d L^{*} / L^{*}\right)$. La pendiente de la curva OA en el Grafico 1 está dada por $\left(1-\delta_{3}\right) \delta_{1}(w / p) / L$.

Igualando $(\mathrm{OA})$ y $(\mathrm{DA})$ se obtiene que

(21) $\frac{d L}{L}=\frac{-\left(1-\delta_{3}\right) \delta_{2}}{\frac{1}{R}-\left(1-\delta_{3}\right) \delta_{1}} \frac{d L^{*}}{L^{*}}$

Es decir, que la variación porcentual del empleo agregado es negativa ante un incremento porcentual de la fuerza laboral si la pendiente de la curva DA es mayor que la pendiente de la curva OA. Esta condición es

(22) $\frac{1}{R}>\left(1-\delta_{3}\right) \delta_{1}$

Igualmente, la variación porcentual de los salarios reales, que es negativa ante un incremento porcentual de la fuerza laboral, está dada por

(23) $\frac{d \frac{w}{p}}{\frac{w}{p}}=-\left(1-\delta_{3}\right) \delta_{2}\left(\frac{\delta_{1}\left(1-\delta_{3}\right)}{\frac{1}{R}-\left(1-\delta_{3}\right) \delta_{1}}+1\right) \frac{d L^{*}}{L^{*}}$

El sistema DA-OA se puede expresar como

$$
\begin{aligned}
& \text { (DA) }-R \frac{d \frac{w}{p}}{\frac{w}{p}}+\frac{d L}{L}=0 \\
& \text { (OA) } \frac{-1}{1-\delta_{3}} \frac{d \frac{w}{p}}{\frac{w}{p}}+\delta_{1} \frac{d L}{L}=-\delta_{2} \frac{d L^{*}}{L^{*}}
\end{aligned}
$$


Las condiciones de estabilidad son que el determinante de este sistema DA-OA sea positivo y que la traza sea negativa. La condición (22) asegura que el determinante del sistema DA-OA es positivo. Esto es, que $-R \delta_{1}+1 / 1-\delta_{3}>0$. Y si $R>\delta_{1}$, esto es, si $1 / \delta_{1}>1 / R$, la traza de este mismo sistema es negativa $\left(-R+\delta_{1}<0\right)$.

\section{REFERENCIAS BIBLIOGRÁFICAS}

Altamirano, Teófilo (2010). Los peruanos en el exterior y su revinculación con el Perú. Lima: Academia Diplomática, Ministerio de Relaciones Exteriores del Perú.

Blanchflower, David y Adam Posen (2014). Wages and Labor Market Slack: Making the Dual Mandate Operational. Working Paper 14-6, Peterson Institute for International Economics.

Borjas, George (2009). The Analytics of the Wage Effect of Inmigration. Working Paper 14796, National Bureau of Economic Research.

Borjas, George J. y Abdurrahman Aydemir (2006). A Comparative Analysis of the Labor Market Impact of International Migration: Canada, Mexico and the United States. Working Paper 12327, National Bureau of Economic Research.

Dancourt, Oscar (1990). Notas sobre desempleo y pobreza en Lima Metropolitana. En Marcel Valcárcel (ed.), Pobreza urbana. Relaciones económicas y marginalidad religiosa (pp. 13-58). Lima: Facultad de Ciencias Sociales, Pontificia Universidad Católica del Perú.

Freyre Paulet, Jorge (2018). Los riesgos del permiso temporal de permanencia (PTP). Gestión, 2 de febrero, p. 16.

Hatton, Timothy y Jeffrey Williamson (2005). Global Migration and the World Economy. Two Centuries of Policy and Performance. Cambridge, MA: MIT Press.

Hunt, Jennifer (1992). The Impact of the 1962 Repatriates from Algeria on the French Labor Market. ILR Review, 45(3). https://doi.org/10.2307/2524278

Documento recibido el 10 de mayo de 2018 y aceptado el 10 de julio de 2018 